Research Article

\title{
Stochastic Analysis on the Resonance of Railway Trains Moving over a Series of Simply Supported Bridges
}

\author{
Tao Zhang $\mathbb{D}^{1,2}$ Taimu Jin $\mathbb{D}^{2},^{2}$ Jun Luo $\mathbb{D}^{1},{ }^{1}$ Shengyang Zhu $\mathbb{D}^{1},{ }^{1}$ and Kaiyun Wang $\mathbb{D}^{1}$ \\ ${ }^{1}$ State Key Laboratory of Traction Power, Southwest Jiaotong University, Chengdu 610031, China \\ ${ }^{2}$ National Innovation Center of High Speed Train, Qingdao 266000, China \\ Correspondence should be addressed to Jun Luo; 1964965318@qq.com
}

Received 29 April 2021; Revised 12 October 2021; Accepted 28 October 2021; Published 13 November 2021

Academic Editor: Stefano Marchesiello

Copyright (c) 2021 Tao Zhang et al. This is an open access article distributed under the Creative Commons Attribution License, which permits unrestricted use, distribution, and reproduction in any medium, provided the original work is properly cited.

Resonance problems encountered in vehicle-bridge interaction (VBI) have attracted widespread concern over the past decades. Due to system random characteristics, the prediction of resonant speeds and responses will become more complicated. To this end, this study presents stochastic analysis on the resonance of railway trains moving over a series of simply supported bridges with consideration of the randomness of system parameters. A train-slab track-bridge (TSB) vertically coupled dynamics model is established following the basic principle of vehicle-track-coupled dynamics. The railway train is composed of multiple vehicles, and each of them is built by seven rigid parts assigned with a total of 10 degrees of freedom. The rail, track slab, and bridge are considered as Euler-Bernoulli beams, and the vibration equations of which are established by the modal superposition method (MSM). Except for the nonlinear wheel-rail interaction based on the Hertz contact theory, the other coupling relations between each subsystem are assumed to be linear elastic. The number theory method is employed to obtain the representative sample point sets of the random parameters, and the flow trajectories of probabilities for the TSB dynamics system are captured by a probability density evolution method (PDEM). Numerical results indicate that the maximum bridge and vehicle responses are mainly dominated by the primary train-induced resonant speed; the last vehicle of a train will be more seriously excited when the bridges are set in resonance by the train; the resonant speeds and responses are rather sensitive to the system randomness, and the possible maximum amplitudes predicted by the PDEM are significantly underestimated by the traditional deterministic method; optimized parameters of the TSB system are preliminary obtained based on the representative point sets and imposed screening conditions.

\section{Introduction}

Railway bridges account for a large proportion of the substructures in railway lines due to safety, comfort, and mitigation of noise pollution. For instance, the BeijingShanghai high-speed railway line is $1318 \mathrm{~km}$ long, and the percentage of total bridge length reaches $80.5 \%$. Due to the fact of higher operating speeds and repetitive nature of train loads, one particular issue of concern in the vehicle-bridge interaction (VBI) is the resonance between the two subsystems [1], i.e., the supporting bridge and the moving train. As a result, the amplification of vehicle and bridge vibrations due to the resonance may jeopardize the vehicles' running comfort and safety, which should be of great concern for railway maintainers and bridge designers.
The resonance problem encountered in VBI has received extensive investigations since the 1990s. $\mathrm{Li}$ and $\mathrm{Su}$ [2] studied the resonant vibration of a girder bridge under highspeed trains with the vehicles modelled as a series of moving loads and a rigid body with two degrees of freedom. The relations of resonance speeds to the parameters of the bridge and vehicle were given. Ju and Lin [3] investigated the resonance characteristics of the simply supported bridge, including high piers under high-speed trainloads. They found that a suitable axial stiffness between two simple beams could reduce vibrations at a near-resonance condition. Museros and Alarcón [4] discussed the contribution of the second bending mode to the dynamic behavior of simply supported railway bridges. The reasons that cause the contribution of the second bending mode to be relevant in 
some situations were highlighted, particularly with regard to the computation of the maximum acceleration. Yau and Yang [5] studied the vertical acceleration response of a simple beam traveled by a series of equally spaced moving loads at constant speeds by the superposition method. It is concluded that for a beam properly damped, the maximum acceleration response of the beam is dominated by the fundamental vibration mode. Xia et al. [6] classified the resonant responses of the bridge induced by moving trains into three types according to different resonance mechanisms, and the resonant train speeds for some bridges were estimated and compared with those obtained from the dynamics model or the field measurements. Liu et al. [7] investigated the conditions under which dynamic trainbridge interaction should be considered for the dynamic analysis of a bridge during a train passage. The results of a moving load model were compared with those of an analysis of dynamic train-bridge interaction considering different vehicle models with a varying degree of sophistication. Mao and $\mathrm{Lu}$ [8] developed a new resonance severity indicator, called $Z$ factor, for the assessment of the resonance effect and found that the resonance severity was essentially governed by the ratio between the bridge and carriage lengths. Yang and Yau $[9,10]$ proposed a semianalytical approach to study the resonance of train cars moving over a series of simple beams. The numerical examples indicate that the vertical acceleration induced by pitching resonance dominates the peak response of the train and neglecting the effect of may underestimate slightly the beam response in the high-speed range and less slightly the moving vehicle response. Moliner et al. [11] addressed the dynamic behavior of double track simply supported bridges of short to medium span lengths considering the contribution of transverse vibration modes and the flexibility of the elastomeric bearings. The influence of these two aspects in the verification of the serviceability limit state of vertical accelerations was evaluated. Duan et al. [12] presented dynamic analysis on train-bridge systems considering the coach-coupler effect by means of the vector form intrinsic finite element method. Results show that the couplers play an energy-dissipating role in reducing the car bodies' resonance response and lead to a dual resonance phenomenon. Yau et al. [13] and Stoura and Dimitrakopoulos [14] assessed the additional damping effect on VBI under resonant excitations. The key parameters dominating the additional damping problem were identified [13], and more importantly, it is found that additional damping can obtain negative values, which implies influx instead of dissipation of energy [14]. Recently, Zhai et al. [15] presented a state-of-the-art review on train-track-bridge dynamic interactions and provided further improvement of the dynamic interaction model and the challenging research topics in the future.

A scrutiny of the previous studies on the resonance problem induced by VBI indicates that most of them were performed using the deterministic dynamic analysis method, and random characteristics of the train and bridge systems may have great influence on the resonance and dynamic responses. In recent years, some scholars have been devoted to the implementation of stochastic dynamics theory into VBI analysis. Yu and Ma [16] and Mao et al. [17] conducted stochastic analysis on train-bridge interactions with random system parameters or track geometric irregularity excitation. Xiao et al. [18] further considered the effects of a track structure on the bridge and the randomness of its parameters. Wang et al. [19] presented random dynamic analysis on a high-speed train moving over a longspan cable-stayed bridge. The long-span cable-stayed bridge model is established by the ANSYS software, based on which its frequencies and mode shapes are exported and implemented into the dynamics programme executed in the MATLAB platform. Their studies [16-19] were conducted based on the probability density evolution method (PDEM) developed by $\mathrm{Li}$ and Chen $[20,21]$, which improves the computational efficiency of the traditional Monte-Carlo method and captures the flow trajectories of probabilities for stochastic systems. Apart from the PDEM, Xu et al. [22] combined the Monte-Carlo method (MCM) and Karhunen-Loève expansion (KLE) to model the random field of track-bridge systems. Xin et al. [23] investigated train-induced resonance analysis by introducing a random propagation process into the VBI. The Nataf-transformation-based point estimation method is applied to generate pseudorandom variables following arbitrarily correlated probability distributions. Numerical results show that the critical train speeds associated with resonance and cancellation are random in essence owing to the variability of system parameters, and the influence of track irregularities on the wheel-rail interactions are significantly greater than those of resonance. Despite a number of studies on the stochastic analysis, the main focus placed on train-bridge resonance phenomenon is still inadequate (except for Ref. [23]), and some important information is likely to be overlooked in this regard.

The main purpose of this study was to provide a better understanding of the resonance of railway trains moving over simply supported bridges considering the randomness of system parameters, and a preliminary discussion on an optimal matching relationship of TSB parameters is presented. The outline of this article is given as follows. Section 2 elaborates the theoretical modelling of a vertical train-slab track-bridge (TSB)-coupled dynamics system. The resonance mechanism involving train-induced bridge and bridge-induced train resonant speeds are introduced in Section 3. Section 4 presents the number theory method and probability density evolution method for stochastic analysis. In Section 5, numerical simulation is carried out to illustrate the dynamic characteristics of the coupled system, and some representative conclusions are given in Section 6 .

\section{Train-Slab Track-Bridge-Coupled Dynamics Model}

Figure 1 shows a TSB-coupled dynamics model following the basic theoretical framework of vehicle-track-coupled dynamics [24]. The entire model is composed of a railway train, rails, track slabs, and $32.5 \mathrm{~m}$ simply supported girders, which are coupled together via the wheel-rail, rail-slab, and 


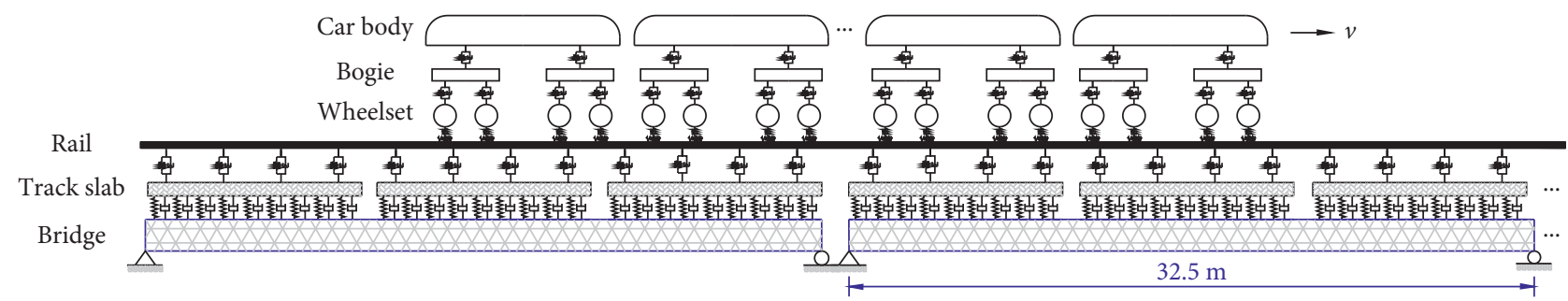

Figure 1: Train-slab track-bridge coupled dynamics model.

slab-bridge interactions. The following basic assumptions are adopted for the dynamics system:

(1) The train moves over the bridge with constant speed $v$, and the coupler and draft gear systems are omitted in the train system, i.e., interaction between adjacent vehicles is not considered

(2) The main focus is placed on the vertical vibrations of the TSB system

(3) Except for the nonlinear wheel-rail interaction based on the Hertz contact theory, the other coupling relations between each subsystem are assumed to be linear elastic

The detailed construction and modelling strategies will be introduced in the following sections.
2.1. EOM of Vehicle. The railway train consists of multiple four-wheelset vehicles, and each vehicle is built by seven rigid parts involving a car body, two bogies, and four wheelsets with a two-stage suspension system. The car body and bogies are, respectively, assigned with two degrees of freedom (DOFs) involving the vertical displacement $Z$ and the pitch angle $\beta$, and each wheelset consists of the DOF of vertical displacement $Z$. Therefore, each vehicle has a total of ten DOFs. The equations of motion (EOM) of the $j$-th vehicle $\left(j=1,2, \ldots, N_{v}\right.$.) can be assembled in the form of second-order differential equations in the time domain:

$$
M_{v j} \ddot{X}_{v j}+C_{v j} \dot{X}_{v j}+K_{v j} X_{v j}=F_{v j},
$$

with

$$
\begin{aligned}
& X_{v j}=\left[\begin{array}{lllllllllll}
Z_{c} & \beta_{c} & Z_{t} & \beta_{t} & Z_{t} & \beta_{t} & Z_{w} & Z_{w} & Z_{w} & Z_{w}
\end{array}\right]^{T}, \\
& M_{v j}=\operatorname{diag}\left\{\begin{array}{llllllllllll}
m_{c} & J_{c} & m_{t} & J_{t} & m_{t} & J_{t} & m_{w} & m_{w} & m_{w} & m_{w}
\end{array}\right\}, \\
& K_{v j}=2 \times\left[\begin{array}{cccccccccc}
2 k_{2 z} & 0 & -k_{2 z} & 0 & -k_{2 z} & 0 & 0 & 0 & 0 & 0 \\
& 2 k_{2 z} L_{c}^{2} & k_{2 z} L_{c} & 0 & -k_{2 z} L_{c} & 0 & 0 & 0 & 0 & 0 \\
& & 2 k_{1 z}+k_{2 z} & 0 & 0 & 0 & -k_{1 z} & -k_{1 z} & 0 & 0 \\
& & & 2 k_{1 z} L_{t}^{2} & 0 & 0 & k_{1 z} L_{t} & -k_{1 z} L_{t} & 0 & 0 \\
& & & & 2 k_{1 z}+k_{2 z} & 0 & 0 & 0 & -k_{1 z} & -k_{1 z} \\
& & & & & 2 k_{1 z} L_{t}^{2} & 0 & 0 & k_{1 z} L_{t} & -k_{1 z} L_{t} \\
& & & & & & k_{1 z} & 0 & 0 & 0 \\
& & & & & & & k_{1 z} & 0 & 0 \\
& & & & & & & & k_{1 z} & 0 \\
\text { sym. } & & & & & & & & & k_{1 z}
\end{array}\right] \text {, } \\
& C_{v j}=2 \times\left[\begin{array}{cccccccccc}
2 c_{2 z} & 0 & -c_{2 z} & 0 & -c_{2 z} & 0 & 0 & 0 & 0 & 0 \\
& 2 c_{2 z} L_{c}^{2} & c_{2 z} L_{c} & 0 & -c_{2 z} L_{c} & 0 & 0 & 0 & 0 & 0 \\
& & 2 c_{1 z}+c_{2 z} & 0 & 0 & 0 & -c_{1 z} & -c_{1 z} & 0 & 0 \\
& & & 2 k_{1 z} L_{t}^{2} & 0 & 0 & c_{1 z} L_{t} & -c_{1 z} L_{t} & 0 & 0 \\
& & & & 2 c_{1 z}+c_{2 z} & 0 & 0 & 0 & -c_{1 z} & -c_{1 z} \\
& & & & & 2 c_{1 z} L_{t}^{2} & 0 & 0 & c_{1 z} L_{t} & -c_{1 z} L_{t} \\
& & & & & & c_{1 z} & 0 & 0 & 0 \\
& & & & & & & c_{1 z} & 0 & 0 \\
& & & & & & & & c_{1 z} & 0 \\
\text { sym. } & & & & & & & & & c_{1 z}
\end{array}\right] \text {, } \\
& F_{v j}=\left[\begin{array}{lllllll}
0 & 0 & 0 & 0 & 0 & 0 & P_{0}-2 F_{w r z 1}(t) P_{0}-2 F_{w r z 2}(t) P_{0}-2 F_{w r z 3}(t) P_{0}-2 F_{w r z 4}(t)
\end{array}\right]^{T} \text {, }
\end{aligned}
$$


where $X_{v j}$ denotes the displacement vector of the $j$-th vehicle; $M_{v j}, C_{v j}$ and $K_{v j}$ denote the mass, damping, and stiffness matrices of the $j$-th vehicle, respectively; $k_{1 z}$ and $k_{2 z}$ are the stiffness of primary and secondary suspensions, respectively; $L_{c}$ and $L_{t}$ are semilongitudinal distance between bogies and wheelsets, respectively; $F_{v j}$ denotes the external force vector of the $j$-th vehicle; $m_{c}, m_{t}$, and $m_{w}$ are the mass of car body, bogie, and wheelset, respectively; $J_{c}, J_{t}$, and $J_{w}$ are the mass moment of inertia of car body, bogie, and wheelset, respectively; $P_{0}$ denotes the static axle load given by $P_{0}=\left(0.25 m_{c}+0.5 m_{t}+m_{w}\right) g$; and $F_{w r z n}$ denotes the wheelrail vertical contact force.

2.2. EOM of Rail. The rail is modelled by a pinned-pinned Euler-Bernoulli beam subjected to dynamic wheel-rail contact forces and fastener supporting forces. The corresponding EOM is given by

$$
\begin{aligned}
E_{r} I_{r} \frac{\partial^{4} Z_{r}(x, t)}{\partial x^{4}}+m_{r} \frac{\partial^{2} Z_{r}(x, t)}{\partial t^{2}}= & -\sum_{i=1}^{N_{f}} F_{r s z i}(t) \delta\left(x-x_{f i}\right) \\
& +\sum_{n=1}^{N_{w}} F_{w r z n}(t) \delta\left(x-x_{w n}\right),
\end{aligned}
$$

where $E_{r} I_{r}$ is the bending stiffness of the rail, $Z_{r}$ is the vertical displacement of the rail, $m_{r}$ is the mass of the rail per unit length, $x_{w n}$ is the time-varying location of the $n$th wheelset, $x_{f i}$ is the location of the $i$ th rail-supporting point, $F_{r s z i}(t)$ is the $i$ th rail-supporting force, $N_{f}$ is the total number of the fasteners, $N_{w}$ is the total number of wheelsets, and $\delta$ is the Dirac's delta function.

By applying the MSM [24],

$$
Z_{r}(x, t)=\sum_{k=1}^{N_{r}} Z_{r k}(x) q_{r k}(t)=\sqrt{\frac{2}{m_{r} L_{r}}} \sum_{k=1}^{N_{r}} \sin \frac{k \pi x}{L_{r}} q_{r k}(t),
$$

the fourth-order partial equation can be further converted into the following second-order ordinary equations:

$$
\ddot{q}_{r k}(t)+\frac{E_{r} I_{r}}{m_{r}}\left(\frac{k \pi}{L_{r}}\right)^{4} q_{r k}(t)=-\sum_{i=1}^{N_{f}} F_{r s z i}(t) Z_{r k}\left(x_{f i}\right)+\sum_{n=1}^{N_{w}} F_{w r z n}(t) Z_{r k}\left(x_{w n}\right) k=1 \sim N_{r},
$$

where $q_{r k}(t)$ is the generalized coordinate describing the vertical motion of the rail, $L_{r}$ is the calculation length of the rail, $N_{r}$ is the truncated number of the rail mode, and $Z_{r k}$ is the mode function of a simply supported beam.
2.3. EOM of Track Slab. The track slab can be regarded as an Euler-Bernoulli beam with free constraints subjected to the vertical dynamic forces induced by rail-slab and slab-bridge interactions. The following governing differential equation can be established:

$$
E_{s} I_{s} \frac{\partial^{4} Z_{s}(x, t)}{\partial x^{4}}+m_{s} \frac{\partial^{2} Z_{s}(x, t)}{\partial t^{2}}=-\sum_{i=1}^{n_{0}} F_{s b z i}(t) \delta\left(x-x_{s i}\right)+\sum_{i=1}^{N_{f}} 2 F_{r s z i}(t) \delta\left(x-x_{f i}\right)
$$

where $E_{s} I_{s}$ is the bending stiffness of the slab, $Z_{s}$ is the vertical displacement of the slab, $m_{s}$ is the mass of the slab per unit length, $n_{0}$ is the total number of supporting points beneath a slab, $x_{s i}$ is the location of the $i$ th slab supporting point, and $F_{s b z i}(t)$ is the $i$ th slab-bridge interaction force.

By applying the MSM [24],

$$
Z_{s}(x, t)=\sum_{k=1}^{N_{s}} Z_{s k}(x) q_{s k}(t)=q_{s 1}(t)+\sqrt{3}\left(1-\frac{2 x}{L_{s}}\right) q_{s 2}(t)+\sum_{k=3}^{N_{s}}\left[\left(\operatorname{ch} \beta_{k} x+\cos \beta_{k} x\right)-C_{k}\left(\operatorname{sh} \beta_{k} x+\sin \beta_{k} x\right)\right] q_{s k}(t),
$$


with

$$
\begin{aligned}
& \beta_{k}= \begin{cases}\frac{4.73004}{L_{s}}, & k=3, \\
\frac{2 k-3}{2 L_{s}} \pi, & k \geq 4,\end{cases} \\
& C_{k}= \begin{cases}0.982502, & k=3, \\
\frac{\operatorname{ch} \beta_{k} L_{s}-\cos \beta_{k} L_{s}}{\operatorname{sh} \beta_{k} L_{s}-\sin \beta_{k} L_{s}} & k \geq 4 .\end{cases}
\end{aligned}
$$

Equation (6) can be converted into the following secondorder ordinary equations:

$$
\ddot{q}_{s k}(t)+\frac{E_{s} I_{s}}{m_{s}} \beta_{k}^{4} q_{s k}(t)=\frac{1}{m_{s} L_{s}}\left[-\sum_{i=1}^{n_{0}} F_{s b z i}(t) Z_{s k}\left(x_{s i}\right)+\sum_{i=1}^{N_{f}} 2 F_{r s z i}(t) Z_{s k}\left(x_{f i}\right)\right] k=1 \sim N_{s} \text {, }
$$

where $q_{s k}(t)$ is the generalized coordinate describing the vertical motion of the slab, $L_{s}$ is the length of the slab, $N_{s}$ is the truncated number of the slab mode, and $Z_{s k}$ is the mode function of a free-free beam.
2.4. EOM of Bridge. The bridge is modelled by a pinnedpinned Euler-Bernoulli beam subjected to dynamic slabbridge supporting forces. The corresponding EOM in the generalized coordinate is given by

$$
\ddot{q}_{b k}(t)+2 \zeta \sqrt{\frac{E_{b} I_{b}}{m_{b}}}\left(\frac{k \pi}{L_{b}}\right)^{2} \dot{q}_{b k}(t)+\frac{E_{b} I_{b}}{m_{b}}\left(\frac{k \pi}{L_{b}}\right)^{4} q_{b k}(t)=\sqrt{\frac{2}{m_{b} L_{b}}} \sum_{i=1}^{n_{0}} F_{s b z i}(t) \sin \frac{k \pi x_{s i}}{L_{b}} k=1 \sim N_{s},
$$

where $q_{b k}(t)$ is the generalized coordinate describing the vertical motion of the bridge, $\zeta, m_{b}, L_{b}$, and $E_{b} I_{b}$ are the damping ratio, per unit length mass, length, and bending stiffness of the bridge, respectively, and $N_{b}$ is the truncated number of the bridge mode. The bridge dynamic displacement in the Cartesian coordinate can be determined by

$$
Z_{b}(x, t)=\sum_{k=1}^{N_{b}} Z_{b k}(x) q_{b k}(t)=\sqrt{\frac{2}{m_{b} L_{b}}} \sum_{k=1}^{N_{b}} \sin \frac{k \pi x}{L_{b}} q_{b k}(t) .
$$

2.5. Interactions between Each Subsystem. The vehicle, rail, track slab, and bridge subsystems are coupled by the wheelrail contact, rail-slab, and slab-bridge interaction forces, which can be calculated by

$$
\begin{aligned}
& F_{w r z n}(t)=\left\{\begin{array}{c}
{\left[\frac{Z_{w n}(t)-Z_{r}\left(x_{w n}, t\right)-Z_{0 n}(t)}{G}\right]^{3 / 2} Z_{w n}(t)-Z_{r}\left(x_{w n}, t\right)-Z_{0 n}(t) \geq 0} \\
0 \quad Z_{w n}(t)-Z_{r}\left(x_{w n}, t\right)-Z_{0 n}(t)<0
\end{array}\right. \\
& F_{r s z i}(t)=k_{p}\left[\sum_{k=1}^{N_{r}} Z_{r k}\left(x_{f i}\right) q_{r k}(t)-\sum_{k=1}^{N_{s}} Z_{s k}\left(x_{f i}\right) q_{s k}(t)\right]+c_{p}\left[\sum_{k=1}^{N_{r}} Z_{r k}\left(x_{f i}\right) \dot{q}_{r k}(t)-\sum_{k=1}^{N_{s}} Z_{s k}\left(x_{f i}\right) \dot{q}_{s k}(t)\right], \\
& F_{s b z i}(t)=k_{b}\left[\sum_{k=1}^{N_{s}} Z_{s k}\left(x_{s i}\right) q_{s k}(t)-\sum_{k=1}^{N_{b}} Z_{b k}\left(x_{s i}\right) q_{b k}(t)\right]+c_{b}\left[\sum_{k=1}^{N_{s}} Z_{s k}\left(x_{s i}\right) \dot{q}_{s k}(t)-\sum_{k=1}^{N_{b}} Z_{b k}\left(x_{s i}\right) \dot{q}_{b k}(t)\right],
\end{aligned}
$$


in which $Z_{w n}(t), Z_{r}\left(x_{w n}, t\right)$, and $Z_{0 n}(t)$ denote the vertical displacement of the $n$th wheel, the vertical rail displacement at the $n$th wheel position, and the vertical track irregularities, respectively; $R_{w}$ represents the rolling radius of the wheel; and $G$ is the wheel-rail contact constant expressed as $G=3.86 R_{\mathrm{w}}^{-0.115} \times 10^{-8} \mathrm{~m} / \mathrm{N}^{2 / 3} ; k_{p}$ and $c_{p}$ are the stiffness and damping of fasteners, respectively; $k_{b}$ and $c_{b}$ are the stiffness and damping of slab-bridge interactions, respectively.

2.6. Numerical Integration Algorithm. A fast explicit numerical integration algorithm, namely, the Zhai method [25] is adopted to solve the developed TSB-coupled dynamics model. This method has been widely used due to its efficiency and simplicity in the dynamic analysis of vehicletrack interactions [24, 26-28]. The dynamic equations for the TSB system can be arranged in a general form as follows:

$$
M \ddot{X}+C \dot{X}+K X=F(\dot{X}, X),
$$

where $M, C$, and $K$ are the mass, damping, and stiffness matrices, respectively; and $F$ denotes the load matrix. form.

The numerical integration algorithm takes the following

For $n=1$,

$$
\left\{\begin{array}{l}
X_{n}=X_{\text {initial }}, \\
\dot{X}_{n}=\dot{X}_{\text {initial }} \\
\ddot{X}_{n}=M^{-1}\left(F-C \dot{X}_{n}-K X_{n}\right) .
\end{array}\right.
$$

For $n=2$,

$$
\left\{\begin{array}{l}
X_{n}=X_{n-1}+\dot{X}_{n-1} \Delta t+0.5 \ddot{X}_{n-1} \Delta t^{2} \\
\dot{X}_{n}=\dot{X}_{n-1}+\ddot{X}_{n-1} \Delta t \\
\ddot{X}_{n}=M^{-1}\left(F-C \dot{X}_{n}-K X_{n}\right) .
\end{array}\right.
$$

For $n \geq 3$,

$$
\left\{\begin{array}{l}
X_{n}=X_{n-1}+\dot{X}_{n-1} \Delta t+(0.5+\psi) \ddot{X}_{n-1} \Delta t^{2}-\psi \ddot{X}_{n-2} \Delta t^{2}, \\
\dot{X}_{n}=\dot{X}_{n-1}+(1+\varphi) \ddot{X}_{n-1} \Delta t-\varphi \ddot{X}_{n-2} \Delta t, \\
\ddot{X}_{n}=M^{-1}\left(F-C \dot{X}_{n}-K X_{n}\right),
\end{array}\right.
$$

where $X, \dot{X}$, and $\ddot{X}$ denote the displacement, velocity, and acceleration of the system, respectively; the subscript "initial" denotes the initial condition; $\Delta t$ is the time step size for numerical integration, which is set to $1 \times 10^{-4} \mathrm{~s} ; \varphi$ and $\psi$ are the independent parameters used for controlling the stability of the algorithm, and $\varphi=\psi=0.5$ is adopted; the subscript $n$ indicates the integration at the time of $n \Delta t$.

\section{Resonant Speeds of a Train Moving over a Series of Bridges}

3.1. Train-Induced Bridge Resonant Speed. For a train modelled as a sequence of vehicles of equal interval $D_{c}$ traveling at speed $v$ over a bridge, an exciting frequency $v / D c$ will be generated. Resonance will be developed on the bridge once the exciting frequency matches any of the bridge frequencies $\left(f_{b}\right)$. Such a phenomenon is referred to as the train-induced resonance on the bridge [10]. The natural frequencies of the studied simply supported bridge can be readily derived as

$$
f_{b}=\frac{1}{2 \pi} \sqrt{\frac{E_{b} I_{b}}{m_{b}}}\left(\frac{k \pi}{L_{b}}\right)^{2}
$$
by

The train-induced resonant speed can then be calculated

$$
v_{b r}=f_{b} D_{c} .
$$

Actually, a subresonance of the acceleration response could also be generated on the bridge as the vehicles pass through the bridge at the speed of $v_{b r}=f_{b} D_{c} / j$, where $j=2$, $3, \ldots$, representing the number of complete cycles of oscillation of the bridge occurring during the passage of two adjacent loads [12].

3.2. Bridge-Induced Train Resonant Speed. For a vehicle moving over a series of simple beams of identical span length $L_{b}$ at speed $v$, the vehicle will encounter repetitive excitations transmitted from the beam with frequency $v / L_{b}$. Resonance will be developed on the vehicle once the exciting frequency coincides with one of the vehicle's frequencies $\left(f_{v}\right)$. Such a phenomenon is referred to as the bridge-induced resonance for train cars [10]. The vertical $\left(f_{v 1}\right)$ and pitching $\left(f_{v 2}\right)$ frequencies of the car body can be obtained as follows:

$$
f_{v 1}=\frac{1}{2 \pi} \sqrt{\frac{\left(4 /\left(1 / 2 k_{1 z}\right)+\left(1 / k_{2 z}\right)\right)}{m_{c}}}=\frac{1}{2 \pi} \sqrt{\frac{8 k_{1 z} k_{2 z}}{\left(2 k_{1 z}+k_{2 z}\right) m_{c}}},
$$

$$
f_{v 2}=\frac{1}{2 \pi} \sqrt{\frac{\left(4 L_{c}^{2} /\left(1 / 2 k_{1 z}\right)+\left(1 / k_{2 z}\right)\right)}{J_{c}}}=\frac{1}{2 \pi} \sqrt{\frac{8 k_{1 z} k_{2 z} L_{c}^{2}}{\left(2 k_{1 z}+k_{2 z}\right) J_{c}}} .
$$

The bridge-induced resonant speed can then be calculated by

$$
\begin{aligned}
& v_{v r}=f_{v 1} L_{b}, \\
& v_{p r}=f_{v 2} L_{b},
\end{aligned}
$$

where $v_{v r}$ and $v_{p r}$ denote bridge-induced vertical and pitching resonant speeds, respectively.

\section{Probability Density Evolution Method}

The random characteristics of the TSB system may have great influence on the dynamic responses. It is assumed that the TSB random system is a conservative system, and the total inflow probability in any domain of the state space is equal to the outflow probability transited through the domain boundary, i.e., no new random factors arise or vanish 
in the process of probability density evolution. Based on the principle of probability conservation, an PDEM is applied to capture the flow trajectories of probabilities for the TSB dynamics system. This section is divided into two parts: one is the determination of the representative point sets of random parameters of the TSB system, and the other is the solution to the random dynamic responses of the TSB system based on the PDEM. Monograph [20] can be referred to for more details about the methodology and solution.

4.1. Representative Point Sets of Random Parameters. In the random vibration analysis, it is unavoidable to encounter the problem of random combinations of different variables. However, Monte-Carlo simulation (MCS) is proved to be time consuming and is also difficult to obtain the timedependent responses of the system. To this end, the number theory method (NTM) $[29,30]$ is employed to obtain the representative sample point sets of the random parameters. Based on the NTM, a uniformly scattered point set in the unit hypercube $[0,1]^{s}$ can be determined by an integer vector:

$$
x_{k, j}=\frac{h_{j} k}{n}-\operatorname{int}\left(\frac{h_{j} k}{n}\right)
$$

where $k=1,2, \ldots, s, s$ denotes the total number of random variables; int $(\cdot)$ denotes the integer part of the value; $j=1,2, \ldots, n,\left(n, h_{1}, h_{2}, \ldots, h_{s}\right)$ is the integer generator vector.

As the total vector number $n$ in the points set is too large, a sphere sieving hypercube method [20] is employed to sieve the points set for higher computational efficiency.

$$
\sum_{k=1}^{s}\left[2\left(x_{k, q}-0.5\right)\right]^{2} \leq r_{0}^{2}\left(1 \leq r_{0}<\sqrt{s}\right)
$$

where $r_{0}$ is the sieving radius, $q=1,2, \ldots, n_{\text {sel }}, n_{\text {sel }}$ is the total remaining number of representative points after sieving $\left(n_{\text {sel }}<<n\right)$.

Considering the difference between the distribution domain of actual random parameters and the sieving unit point set $x_{k, q}$, the following affine transformation is performed:

$$
\xi_{k, q}=\mu_{k}\left[1+2 \lambda \nu_{k}\left(x_{k, q}-0.5\right)\right],
$$

where $\lambda$ is the truncated boundary, $\mu_{k}$ is the mean value of the random variable, and $v_{k}$ is the variation coefficient of the random variable.

After obtaining the representative sample point sets $\xi_{q}$ for multidimensional random parameters, the probabilistic space $\Omega_{\Theta}$ of the random samples is then be divided into Voronoi regions with representative volumes $V\left(\xi_{q}\right)$ satisfying

$$
\bigcup_{q}^{n_{\text {sel }}} V\left(\xi_{q}\right)=\Omega_{\Theta,} V\left(\xi_{i}\right) \cap V\left(\xi_{j}\right), \quad i \neq j .
$$
by

The initial probability of each designed point set is given

$$
P_{q}=\int_{V\left(\xi_{q}\right)} p_{\Theta}\left(\xi_{q}\right) d \xi
$$

where $p_{\Theta}\left(\xi_{q}\right)$ denotes the probability density of the random parameters in Voronoi region $V\left(\xi_{q}\right)$, and the total probability satisfies the following equation:

$$
\sum_{q=1}^{n_{\text {sel }}} P_{q}=\sum_{q=1}^{n_{\text {sel }}} \int_{\xi_{q}} p_{\Theta}\left(\xi_{q}\right) d \xi=\int_{\substack{n_{\text {sel }} \\ q=1}} p_{q}\left(\xi_{q}\right) d \xi=\int_{\Omega_{\Theta}} p_{\Theta}\left(\xi_{q}\right) d \xi=1 .
$$

4.2. Solution with PDEM. Through introducing state vector $U=\left(X^{T}, \dot{X}^{T}\right)^{T}$ obtained by solving equation (13), the state vector can be further expressed as the augmented state vector

$$
Y=\left(U^{T}, \xi_{q}^{T}\right)^{T}
$$

Denote the joint PDF of $Y(t)$ as $p_{Y}(y, t), y=\left(y_{1}, \ldots, y_{2 n}\right.$, $\left.\mathrm{y}_{\xi}^{\mathrm{T}}\right)^{\mathrm{T}}$, in which $n$ is the number of DOF in the TSB system. By considering the TSB system as a probability conservative system, the probability density equation is deduced as follows [21]:

$$
\begin{gathered}
\frac{\partial}{\partial t} p_{Y}(y, t)+\sum_{i=1}^{2 n} \frac{\partial}{\partial y_{i}} g_{i}\left(y_{\xi}, t\right) p_{Y}(y, t)=0 \\
\text { with } g_{i}=\frac{\partial}{\partial t} U_{i}(\xi, t)
\end{gathered}
$$

The initial condition of equation (29) can be expressed as follows:

$$
p_{U_{l \Theta}}\left(u_{l}, \xi_{q}, \quad t_{0}\right)=\delta\left(u_{l}-U_{l, 0}\right) p_{\Theta}\left(\xi_{q}\right) 1 \leq l \leq 2 n,
$$

where $U_{l, 0}$ is the initial value of $u_{l}$.

The total deviation diminishing (TVD) difference scheme is applied to solve the partial differential equations jointed by equations (29) and (30), and the joint probability function $p_{U l \Theta}\left(u_{l}, \xi_{q}, t\right)$ can be solved. Then, an integration of $\xi_{q}$ is performed to obtain probability density function of $u_{l}$ :

$$
p_{U_{l}}\left(u_{l}, t\right)=\int p_{U_{l \Theta}}\left(u_{l}, \xi_{q}, t\right) d \xi .
$$

\section{Model Validation}

This section will illustrate the validity of the established TSB dynamics model by comparing resonant responses derived by those of Yang and Yau [10], who developed a VBI element considering the rail effect. Consider a train composed of twelve vehicles moving over six simply supported bridges. All the calculation parameters can be found in the study by Yang and Yau [10]. Figure 2 demonstrates the comparison of maximum midpoint accelerations of the bridge with various train speeds. It can be clearly seen that the two models are basically in good agreements. Some marginal differences can be attributed to the different modelling methods (FEM and 


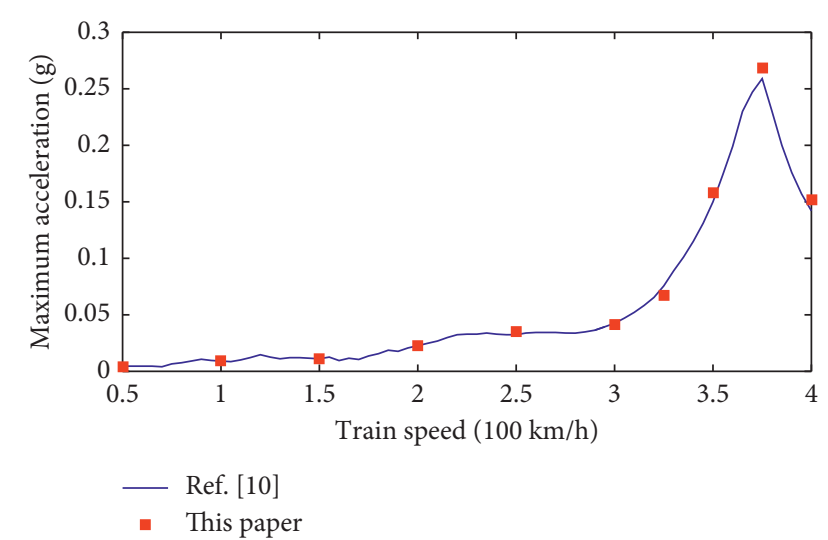

FIgURE 2: Comparisons of bridge acceleration versus train speed obtained from this model and from the study by Yang and Yau [10].

MSM) and the neglect of slab tracks referred in the study by Yang and Yau [10]. Overall, the comparison results have properly illustrated the reliability of the proposed dynamics model, which will be employed in the follow-up study.

\section{Numerical Application}

6.1. General Information. Consider a train composed of eight vehicles moving over five-span simply supported bridges under the excitation of track irregularities. The sample of track vertical irregularities characterized by wavelengths between 1 and $120 \mathrm{~m}$ is adopted in the simulation, as displayed in Figure 3. The mechanical parameters of railway vehicle, track, and bridge are listed in Table 1. In addition, the random variables in the TSB system are assumed to obey the normal distribution with the coefficients of variation (COVs) listed in Table 2.

6.2. Deterministic Analysis on the Resonance. In order to compare with the follow-up stochastic analysis and ascertain the matching relations between resonant speeds and dynamic responses, a deterministic study will be conducted first. According to the vehicle and bridge parameters listed in Table 1, the resonant speeds can be calculated based on equations (17)-(21), as listed in Table 3 . The maximum acceleration of the car body $a_{\mathrm{vmax}}$ is defined as follows [9].

$$
a_{v \max }=\max \left(\left|\ddot{Z}_{c} \pm \frac{D_{c} \ddot{\beta}_{c}}{2}\right|\right),
$$

where $\ddot{Z}_{c}$ and $\ddot{\beta}_{c}$ are the vertical and rotational accelerations of the centroid of car body, respectively.

The train speed varies from 50 to $400 \mathrm{~km} / \mathrm{h}$ with an increment of $10 \mathrm{~km} / \mathrm{h}$ that is small enough to demonstrate the maximum dynamic responses of both bridge and train if resonance occurs. The maximum accelerations of the bridge $\left(a_{b \max }\right)$ against the train speed are illustrated in Figure 4 . As can be seen, there exist a significant resonant peak $(0.17 \mathrm{~g})$ at the speed of $340 \mathrm{~km} / \mathrm{h}$ and a subresonant peak at the speed of $110 \mathrm{~km} / \mathrm{h}$, which are very close to the primary resonant speed $v_{b r 1}=346 \mathrm{~km} / \mathrm{h}$ and $v_{b r 1} / 3$. In Figure 5, the bridge midpoint acceleration with the primary resonant speed $v_{b r}$

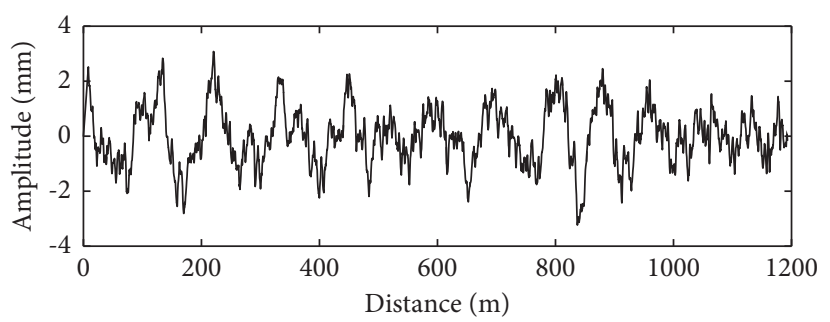

FIgURE 3: Sample of track vertical irregularities adopted in the simulation.

and pitching resonance speed $v_{p r}$ are compared. It can be seen that the bridge acceleration continues to increase when the train travels at the primary resonant speed, $v_{b r}$, and the amplitude is much larger than that with the pitching resonant speed $v_{p r}$. Although the car body vibrations will be obviously excited by the pitching resonant speed as shown in Figure 6, its influence will not be reflected on the bridge. One can observe from Figure 7 that the bridge acceleration increases considerably near the frequency of $3.88 \mathrm{~Hz}$ at the train speed of $346 \mathrm{~km} / \mathrm{h}$. This can be explained by the fact that when the train runs with the speed of $346 \mathrm{~km} / \mathrm{h}$, and the dominant frequency of the train loading is $v / D c=3.88 \mathrm{~Hz}$, which approaches to the bridge fundamental natural frequency. Besides, a slight amplification can also be found at the intersection of the curve $f_{\mathrm{b}}$ and the curve $3 v / D c$.

Figure 6 illustrates the maximum accelerations of the car body against the train speed. It is found that there exist two clear resonant peaks in the vicinity of 100 and $340 \mathrm{~km} / \mathrm{h}$, corresponding to the bridge-induced pitching resonant speed and the train-induced resonant speed, respectively. Compared with the response amplitude at the vertical resonant speed $v_{v r}$, the resonant responses are dominated by pitching resonant speed. Time histories of the first and last car body accelerations at the train-induced resonant speed are displayed in Figure 8, from which one can deduce that when the bridges are set in resonance by the train, and the last car of the train will be more seriously excited than the front cars by the bridges that were continuously excited by the front passing vehicles. The maximum acceleration of the last vehicle is found to be $0.031 \mathrm{~g}$, which is 1.48 times as large as that for the first one. This amplification phenomenon has also been reported in the study by Yang and Yau [10] previously.

6.3. Stochastic Analysis on the Resonance. The effect of system random characteristics on TSB dynamic interactions will be investigated in this section. In the stochastic analysis, the number of representative point sets after sieving is $n_{\text {sel }}=144$ with the sieving radius of $r_{0}^{2}=1.2$. In other words, a total of 144-point sets are considered for the system random parameters $\left(m_{c}, J_{c}, k_{1 z}, k_{2 z}, c_{1 z}, c_{2 z}, k_{p}, c_{p}, m_{b}, E_{b}, \zeta\right)$. According to the numerical results in Section 6.2, although the amplification effect of dynamic responses will occur in the vicinity of subresonant speeds and bridge-induced resonant speeds, the maximum amplitudes of both bridge and vehicle are actually dominated by the primary train- 
TABLE 1: Mechanical parameters of the railway vehicle, track, and bridge adopted in the simulation.

\begin{tabular}{|c|c|c|c|}
\hline Para & Values & Parameters & Values \\
\hline $\begin{array}{l}\text { Mass of car body } m_{c} \\
\text { Mass of bogie } m_{t} \\
\text { Mass of wheelset } m_{w} \\
\text { Mass moment of inertia of car body } J_{c} \\
\text { Mass moment of inertia of bogie } J_{t} \\
\text { Semilongitudinal distance between bogies } L_{c} \\
\text { Semilongitudinal distance between wheelsets } L_{t} \\
\text { Length of the car body } D_{c} \\
\text { Stiffness of the primary suspension } k_{1 z} \\
\text { Stiffness of the secondary suspension } k_{2 z} \\
\text { Damping of the primary suspension } c_{1 z} \\
\text { Damping of the secondary suspension } c_{2 z} \\
\text { Rolling radius of the wheel } R_{w} \\
\text { Elastic modulus of the rail } E_{r}\end{array}$ & $\begin{array}{l}38.9 \mathrm{t} \\
2.2 \mathrm{t} \\
1.52 \mathrm{t} \\
1905 \mathrm{t} \mathrm{m}^{2} \\
1.233 \mathrm{t} \mathrm{m}^{2} \\
8.6875 \mathrm{~m} \\
1.25 \mathrm{~m} \\
24.775 \mathrm{~m} \\
887 \mathrm{kN} / \mathrm{m} \\
203 \mathrm{kN} / \mathrm{m} \\
10 \mathrm{kN} \mathrm{s} / \mathrm{m} \\
10 \mathrm{kN} \mathrm{s} / \mathrm{m} \\
0.46 \mathrm{~m} \\
2.1 \times 10^{5} \mathrm{MPa}\end{array}$ & $\begin{array}{c}\text { Moment of inertia of the rail cross section } I_{r} \\
\text { Mass of the rail per unit length } m_{r} \\
\text { Stiffness of the rail pad } k_{p} \\
\text { Damping of the rail pad } c_{p} \\
\text { Fastener spacing } L_{f} \\
\text { Elastic modulus of the track slab } E_{s} \\
\text { Moment of inertia of the slab cross section } I_{s} \\
\text { Length/width/thickness of the slab } L_{s} / W_{s} / H_{s} \\
\text { Density of the track slab/bridge } \rho_{s} / \rho_{b} \\
\text { Length of the bridge } L_{b} \\
\text { Mass of the bridge per unit length } m_{b} \\
\text { Elastic modulus of the bridge } E_{b} \\
\text { Moment of inertia of the bridge cross section } I_{b} \\
\text { Damping ratio of the bridge } \zeta\end{array}$ & $\begin{array}{l}3.217 \times 10^{-5} \mathrm{~m}^{4} \\
60.64 \mathrm{~kg} / \mathrm{m} \\
5.0 \times 10^{7} \mathrm{~N} / \mathrm{m} \\
3.6 \times 10^{4} \mathrm{~N} \mathrm{~s} / \mathrm{m} \\
0.65 \mathrm{~m} \\
3.65 \times 10^{4} \mathrm{MPa} \\
1.372 \times 10^{-3} \mathrm{~m}^{4} \\
6.5 / 2.5 / 0.20 \mathrm{~m} \\
2500 \mathrm{~kg} / \mathrm{m}^{3} \\
32.5 \mathrm{~m} \\
31.4 \mathrm{t} / \mathrm{m} \\
3.45 \times 10^{4} \mathrm{MPa} \\
6.2 \mathrm{~m}^{4} \\
0.03\end{array}$ \\
\hline
\end{tabular}

TABLE 2: Coefficients of variation of the random system parameters.

\begin{tabular}{|c|c|c|c|}
\hline Parameters & COVs & Parameters & COVs \\
\hline Mass of car body $m_{c}$ & 0.25 & Stiffness of the rail pad $k_{p}$ & 0.35 \\
\hline Mass moment of inertia of car body $J_{c}$ & 0.25 & Damping of the rail pad $c_{p}$ & 0.35 \\
\hline Stiffness of the primary suspension $k_{1 z}$ & 0.25 & Mass of the bridge per unit length $m_{b}$ & 0.05 \\
\hline Stiffness of the secondary suspension $k_{2 z}$ & 0.25 & Elastic modulus of the bridge $E_{b}$ & 0.25 \\
\hline Damping of the primary suspension $c_{1 z}$ & 0.25 & Damping ratio of the bridge $\zeta$ & 0.25 \\
\hline Damping of the secondary suspension $c_{2 z}$ & 0.25 & & \\
\hline
\end{tabular}

TABLE 3: Train-induced and bridge-induced resonant speeds.

\begin{tabular}{lc}
\hline Characteristic frequencies & $\begin{array}{c}\text { Resonant } \\
\text { speeds }\end{array}$ \\
\hline Vertical frequency $f_{v 1}=0.69 \mathrm{~Hz}$ & $v_{v r}=81 \mathrm{~km} / \mathrm{h}$ \\
Pitching frequency $f_{v 2}=0.86 \mathrm{~Hz}$ & $v_{p r}=101 \mathrm{~km} / \mathrm{h}$ \\
The first natural frequency of the bridge & $v_{b r}=346 \mathrm{~km} / \mathrm{h}$ \\
$f_{b 1}=3.88 \mathrm{~Hz}$ & \\
\hline
\end{tabular}

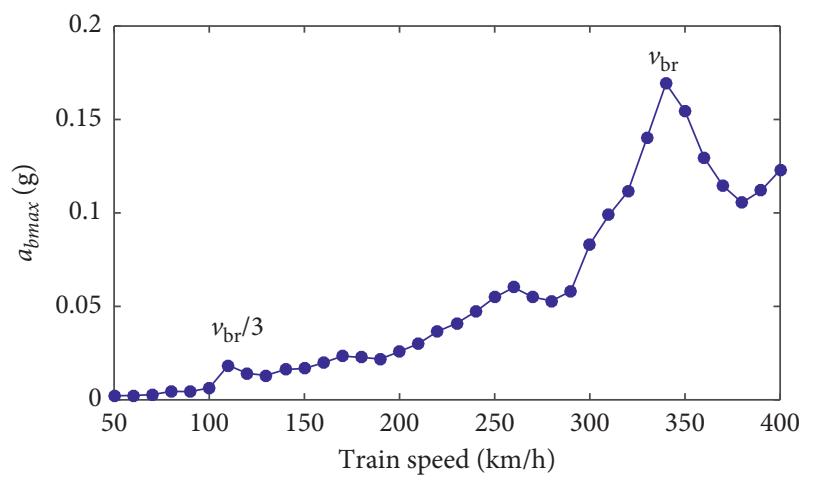

Figure 4: Maximum midpoint accelerations of the bridge versus train speed.

induced resonant speed. Analogous phenomena can also be found in earlier studies $[9,10,12]$. In addition, higher speeds tend to result in more intense wheel-rail interactions, and thus, system vibrations will be amplified accordingly. As

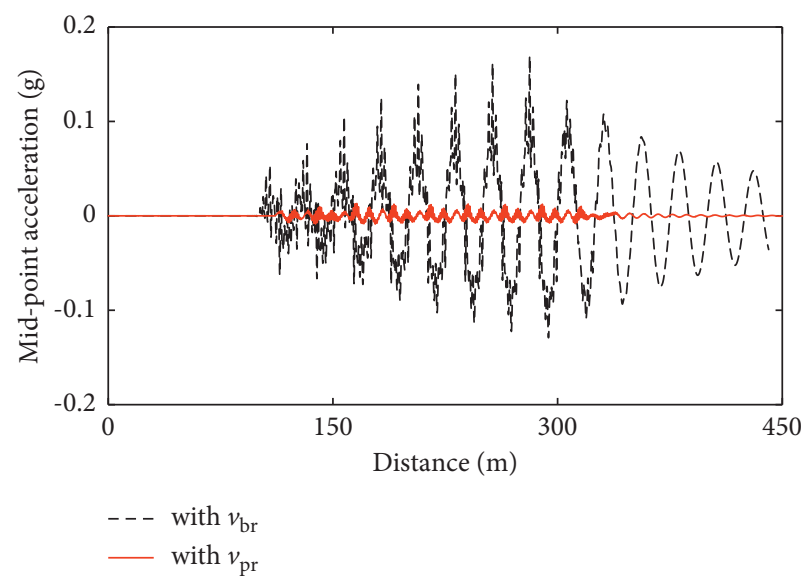

Figure 5: Time histories of bridge midpoint acceleration.

such, our main focus is placed on the operating speed ranging from $300 \mathrm{~km} / \mathrm{h}$ to $400 \mathrm{~km} / \mathrm{h}$ with an increment of $10 \mathrm{~km} / \mathrm{h}$.

The contour of probability density functions (PDF) of bridge and vehicle dynamic responses for $v=340 \mathrm{~km} / \mathrm{h}$ that dominates system responses in the deterministic study are illustrated in Figures 9-11, and the dynamic responses in the first second are omitted. Clearly, the contours are mostly contained within the upper and lower limits (Mean \pm 3 Std), where the mean value curves (mean) and the standard deviation curves (Std) are calculated by the probability functions based on the assumption of a normal distribution. 


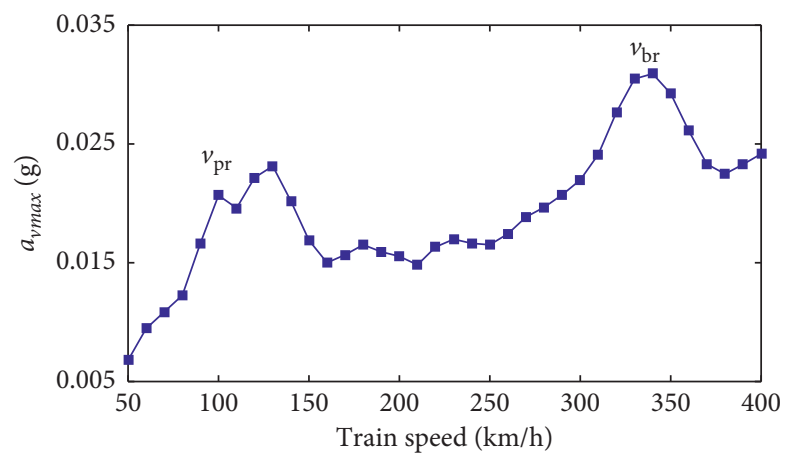

FIGURE 6: Maximum car body accelerations versus train speed.
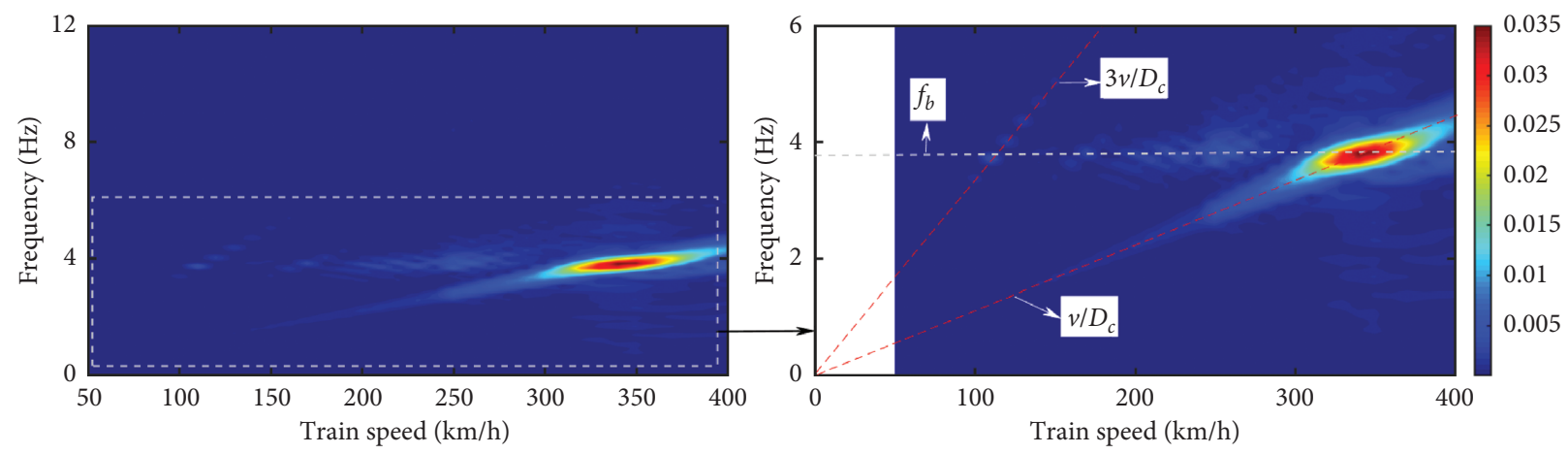

FIGURE 7: Bridge midpoint acceleration in the frequency-domain with various train speeds.

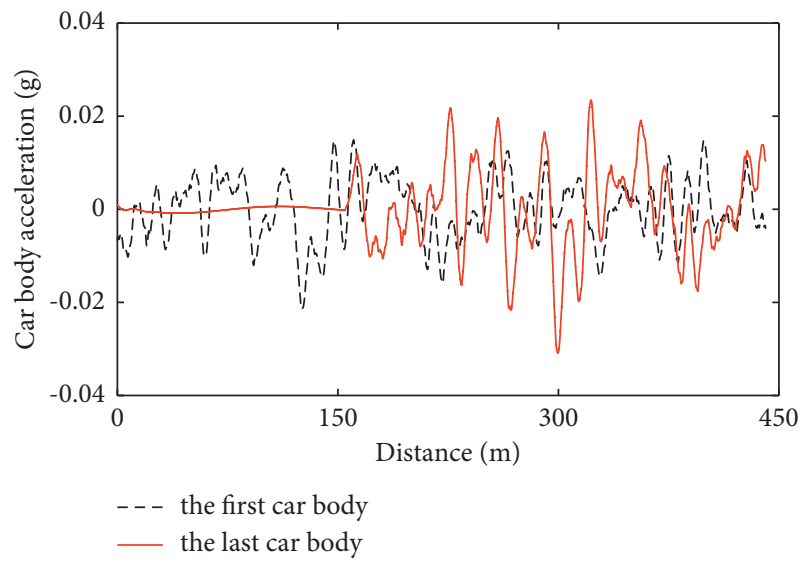

Figure 8: Time histories of the first and last car body accelerations with $(v)_{b r}$.

The solid lines marked as "mean \pm 3 Std" indicate the upper and lower bounds of the probability density functions with a probability guarantee rate of $99.7 \%$. The results obtained by the traditional deterministic method are also shown in the figures, in which all of the stochastic parameters are represented by the mean values. Significant differences can be observed between the results based on the two calculation methods. For instance, the maximum bridge displacement and acceleration are found to be $4.51 \mathrm{~mm}$ and $0.302 \mathrm{~g}$, respectively, which increase by $45.0 \%$ and $78.7 \%$ compared with the deterministic analysis. The car body acceleration reaches the maximum value of $0.053 \mathrm{~g}$, which is 1.71 times as large as that with the deterministic analysis. This confirms that the system randomness greatly affects the dynamic responses, and the traditional deterministic method may fail to estimate the underlying risks due to the neglect of randomness of system parameters.

Moreover, the statistics of the bridge and vehicle maximum accelerations with different train speeds and deviations between different calculation methods are summarized in Tables 4 and 5. It can be seen that the prediction of resonant speed will become complicated when the system randomness is considered. The peak responses do not occur at the resonant speed in the deterministic analysis. With the train speed varying from $300 \mathrm{~km} / \mathrm{h}$ to $400 \mathrm{~km} / \mathrm{h}$, the maximum bridge acceleration is found to be $0.349 \mathrm{~g}$ at $v=380 \mathrm{~km} / \mathrm{h}$, and the maximum car body acceleration is found to be $0.066 \mathrm{~g}$ at $v=400 \mathrm{~km} / \mathrm{h}$. The possible maximum amplitudes predicted by the PDEM are significantly underestimated by the traditional deterministic method.

It should be noted that the Eurocode [31] specified four bridge parameters to perform conservative dynamic analyses in the design phase, involving the lower bound of damping, the lower bound of stiffness, the upper and lower bounds of mass. However, for such a large-scale TSB system with numerous random parameters, it will be hard to determine the most unfavorable parameter combination, which can be efficiently overcome by stochastic 

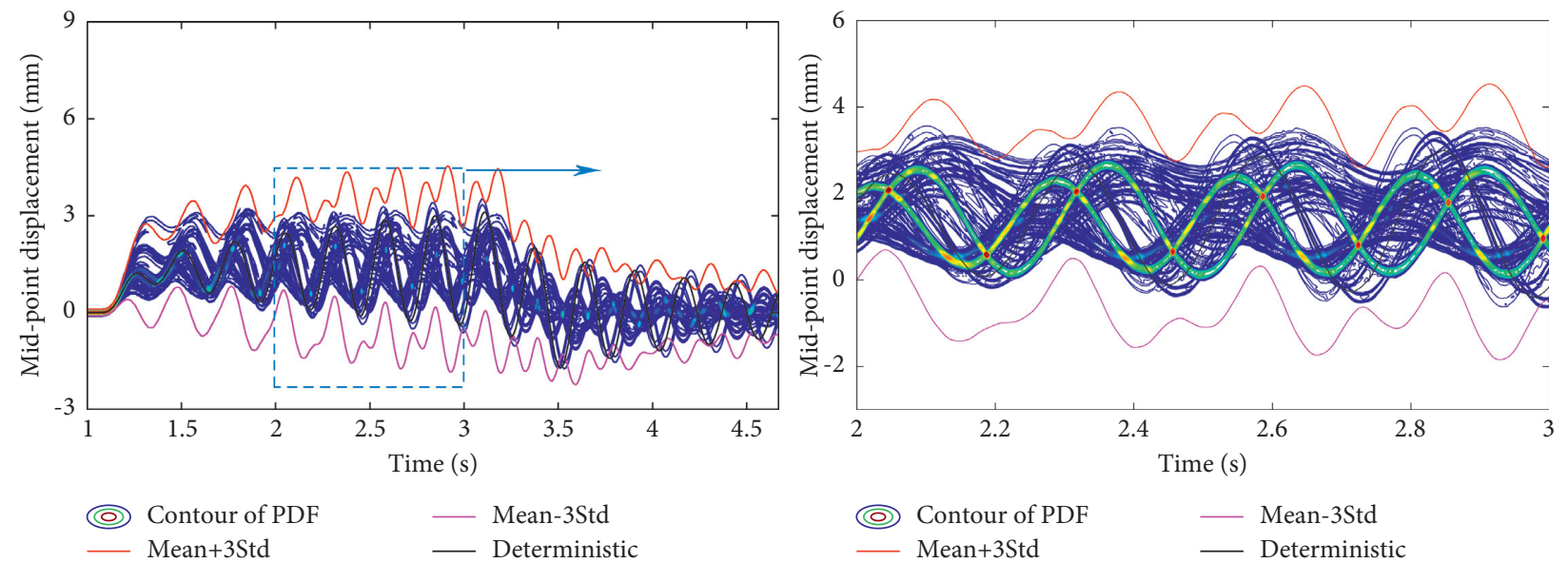

Figure 9: Contour of PDF of bridge midpoint displacement for $(v)=340 \mathrm{~km} / \mathrm{h}$.
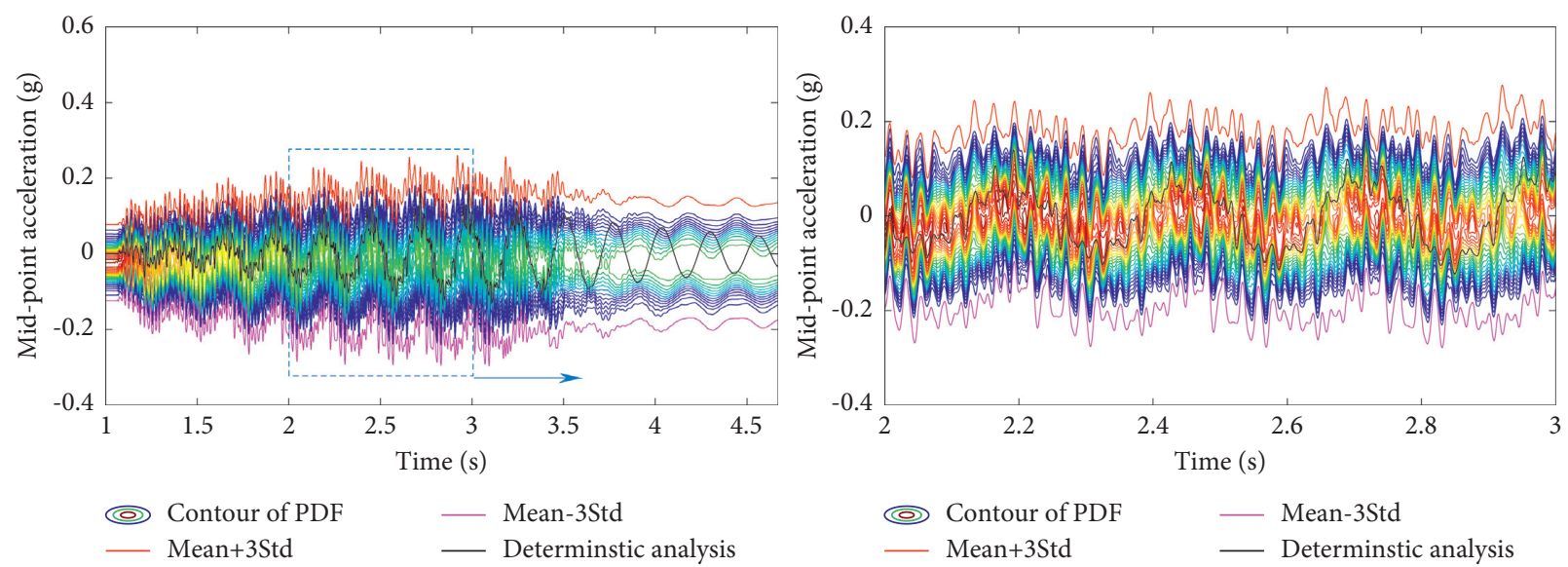

FIgURE 10: Contour of PDF of bridge midpoint acceleration for $(v)=340 \mathrm{~km} / \mathrm{h}$.
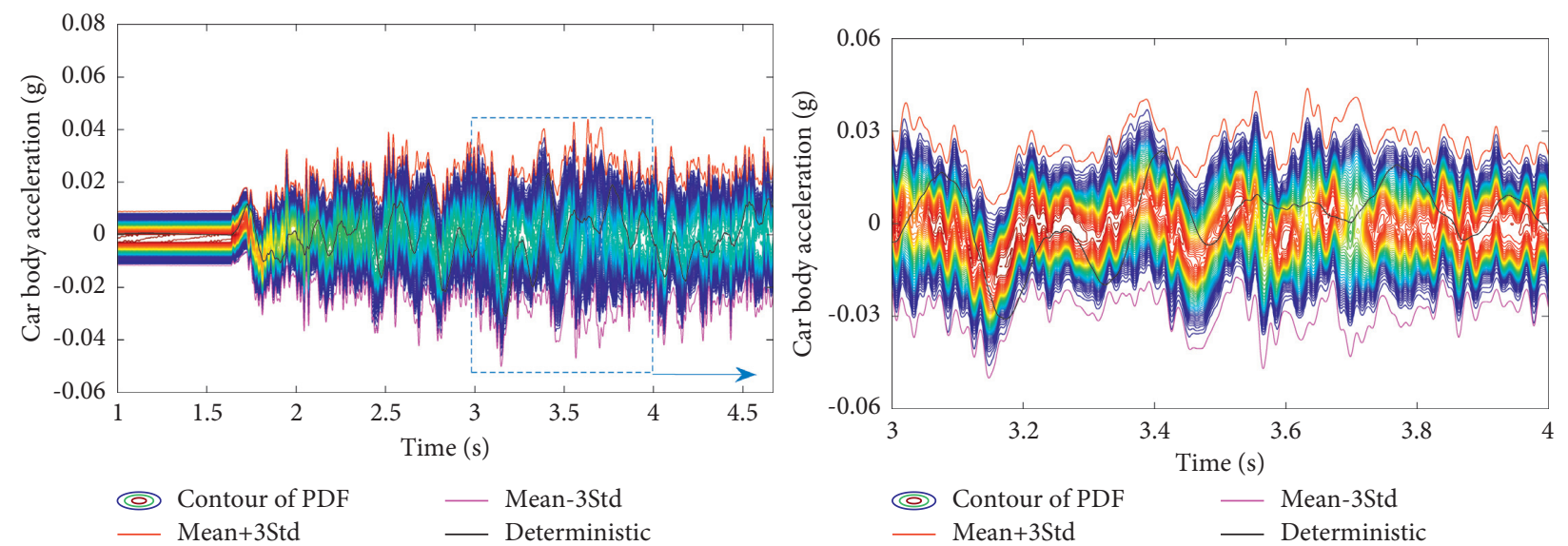

FIGURE 11: Contour of PDF of car body acceleration for $(v)=340 \mathrm{~km} / \mathrm{h}$.

analysis based on PDEM. To sum up, we should not perform deterministic analysis with the mean properties in the bridge design phase, and parameter randomness should be fully considered.
Then, the influence of system randomness on resonant speeds and responses is studied. Based on the 144 representative point sets, the primary train-induced resonant speeds can be obtained for each parameter combination, as 
TABLE 4: Statistics of bridge midpoint maximum acceleration with different train speeds and calculation methods.

\begin{tabular}{lccc}
\hline Train speed $(\mathrm{km} / \mathrm{h})$ & & \multicolumn{2}{c}{$\begin{array}{c}\text { Calculation method } \\
\text { Deterministic analysis }(\mathrm{g})\end{array}$} \\
\hline 300 & 0.241 & 0.083 & 190.4 \\
310 & 0.265 & 0.099 & 167.7 \\
320 & 0.278 & 0.111 & 150.5 \\
330 & 0.297 & 0.140 & 112.1 \\
340 & 0.302 & 0.169 & 78.7 \\
350 & 0.333 & 0.155 & 114.8 \\
360 & 0.343 & 0.130 & 163.8 \\
370 & 0.341 & 0.114 & 199.1 \\
380 & 0.349 & 0.106 & 229.2 \\
390 & 0.346 & 0.112 & 208.9 \\
400 & 0.337 & 0.123 & 174.0 \\
\hline
\end{tabular}

TABLE 5: Statistics of car body maximum acceleration with different train speeds and calculation methods.

\begin{tabular}{lccc}
\hline Train speed $(\mathrm{km} / \mathrm{h})$ & \multicolumn{2}{c}{$\begin{array}{c}\text { Calculation method } \\
\text { Deterministic analysis }(\mathrm{g})\end{array}$} \\
\hline 300 & 0.050 & 0.022 & 127.3 \\
310 & 0.051 & 0.024 & 112.5 \\
320 & 0.048 & 0.028 & 71.4 \\
330 & 0.050 & 0.030 & 66.7 \\
340 & 0.053 & 0.031 & 71.0 \\
350 & 0.057 & 0.029 & 96.6 \\
360 & 0.059 & 0.026 & 126.9 \\
370 & 0.059 & 0.023 & 156.5 \\
380 & 0.059 & 0.022 & 168.2 \\
390 & 0.063 & 0.023 & 173.9 \\
400 & 0.066 & 0.024 & 175.0 \\
\hline
\end{tabular}

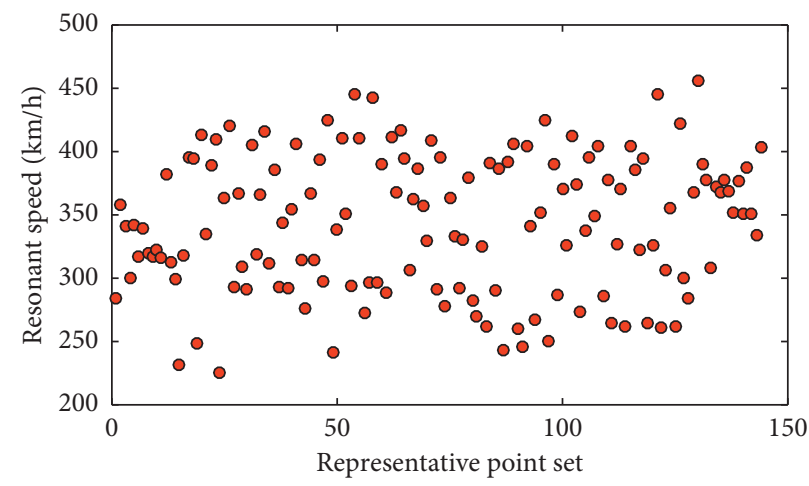

FIGURE 12: Random resonant speeds based on the representative point sets of the TSB system.

shown in Figure 12, from which one can observe that the resonant speeds exhibit a wide range of distribution, varying from $225.7 \mathrm{~km} / \mathrm{h}$ to $455.9 \mathrm{~km} / \mathrm{h}$. Figure 13 illustrates the maximum dynamic responses of the bridge and vehicle excited by the resonant speed in Figure 12. It can be found that the resonant responses have great dispersion. No obvious correlations can be found between the resonant speed and the maximum value of bridge midpoint and car body accelerations, and there exists a negative correlation between the resonant speed and bridge displacement. Furthermore, it can be deduced that the resonance might occur, even though the designed resonant speed has not been reached in operation due to the existence of structural randomness.

There may exist an optimal matching relationship among the bridge, track, and vehicle parameters, so that even if the resonant speed is reached, the corresponding resonant responses will not be amplified obviously or the resonant speed is beyond the operating range. This is an attractive subject that can be carried out in the following study. Here, we only present a preliminary discussion on this issue from 


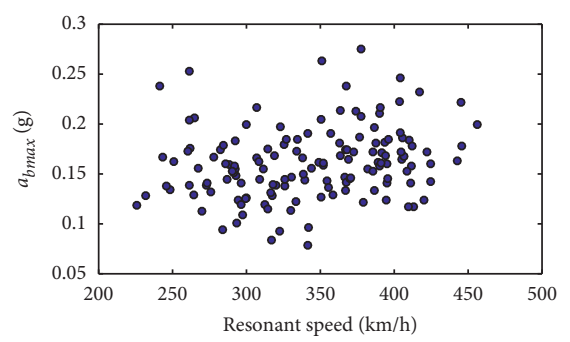

(a)

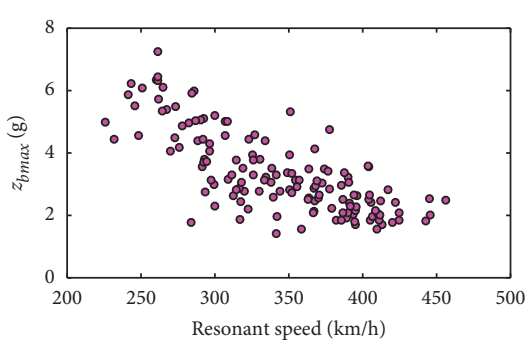

(b)

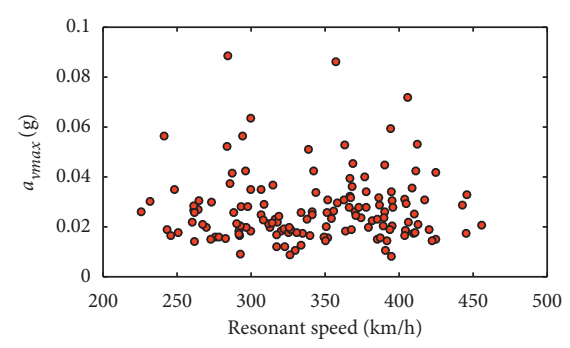

(c)

Figure 13: Maximum dynamic responses versus random train-induced resonant speed: (a) bridge midpoint accelerations; (b) bridge midpoint displacements; (c) car body accelerations.

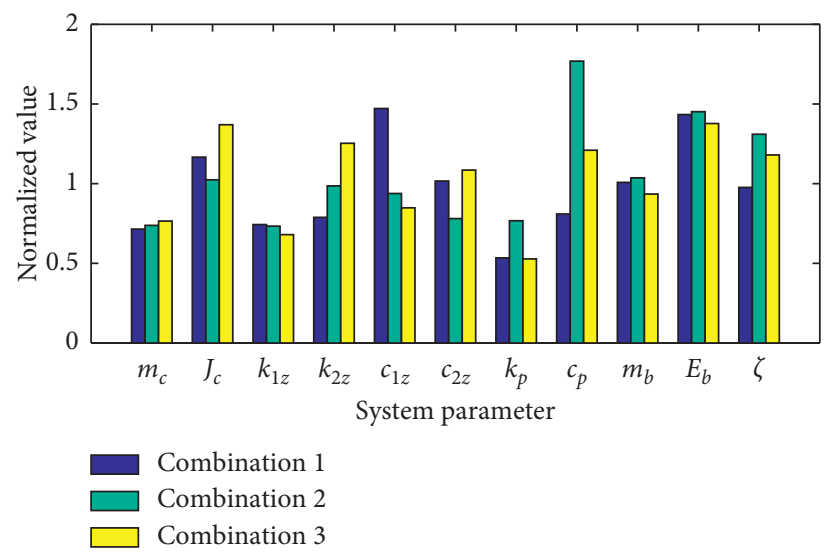

FIGURE 14: Optimized system parameters based on the imposed screening conditions.

the perspective of VBI. The optimized system parameters can be screened by satisfying the following conditions: (1) the resonant speed is large enough that exceeds the practical operating range $v_{0}$, i.e., $v_{b r}>v_{0}$; and (2) and the peak values of resonant responses (denoted by $R_{\text {sto }}$ ) are smaller than those in the deterministic analysis (denoted by $R_{\text {det }}$ ), i.e., $R_{\text {sto }} /$ $R_{\text {det }} \leq \alpha(\alpha<1)$. If $\alpha=0.8$ and $v_{0}=400 \mathrm{~km} / \mathrm{h}$ are adopted, there exist three required combinations of system parameters in the representative point sets displayed in Figure 14, in which all the parameters have been normalized by the mean values listed in Table 1 .

\section{Conclusions}

In this study, stochastic analysis on the resonance of railway trains moving over a series of simply supported bridges is studied by considering system random characteristics. A vertical train-slab track-bridge-coupled dynamics model is established based on the classical vehicletrack coupled dynamics, and random resonance of the system are captured by means of the PDEM. The following conclusions can be reached based on the conducted numerical analysis:

(1) In the deterministic analysis, the maximum amplitudes of both bridge and vehicle responses are mainly dominated by the primary train-induced resonant speed, although the amplification effect of dynamic responses will also occur in the vicinity of subresonant speeds and bridge-induced resonant speeds. When the bridges are set in resonance by the train, the last car of the train will be more seriously excited than the front cars by the bridges that were continuously excited by the front passing vehicles.

(2) The prediction of resonant speed will become complicated when the system randomness is considered. The peak responses do not occur at the expected resonant speed in the deterministic analysis, and resonant speeds exhibit a wide range of distribution, varying from $225.7 \mathrm{~km} / \mathrm{h}$ to $455.9 \mathrm{~km} / \mathrm{h}$ for the cases studied herein. The possible maximum amplitudes predicted by the PDEM are significantly underestimated by the traditional deterministic method, which may fail to capture the underlying risks.

(3) Based on the representative point sets and imposed screening conditions, we obtained optimized parameter combinations of the TSB system. They make the resonant speed exceed $400 \mathrm{~km} / \mathrm{h}$ (the upper limit of practical operation speed), and the maximum dynamic responses involving car body and bridge accelerations and bridge displacement are smaller than those with original mean parameters even if the resonant speed is reached. It is an attractive subject to seek an optimal matching relationship of TSB 
parameters, which deserves in-depth research in future.

\section{Data Availability}

The data used to support the findings of this study are available from the corresponding author upon request.

\section{Conflicts of Interest}

The authors declare that there are no conflicts of interest regarding the publication of this study.

\section{Acknowledgments}

This work was supported by the National Natural Science Foundation of China (11790283, 51978587, and 51778194), the Sichuan Science and Technology Program (2021YFH0073), the Fund from State Key Laboratory of Traction Power (2019TPL-T16), and the 111 Project (B16041), which are gratefully acknowledged by the authors.

\section{References}

[1] Y. Yang, J. Yau, and Y. Wu, Vehicle-bridge Interaction Dynamics-With Applications to High-Speed Railways, World Scientific, Singapore, 2004

[2] J. Li and M. Su, "The resonant vibration for a simply supported girder bridge under high-speed trains," Journal of Sound and Vibration, vol. 224, no. 5, pp. 897-915, 1999.

[3] S. H. Ju and H. T. Lin, "Resonance characteristics of highspeed trains passing simply supported bridges," Journal of Sound and Vibration, vol. 267, no. 5, pp. 1127-1141, 2003.

[4] P. Museros and P. E. Alarcón, "Influence of the second bending mode on the response of high-speed bridges at resonance," J. Struct. Eng.-ASCE.vol. 131, pp. 404-415, 2005.

[5] J. D. Yau and Y. B. Yang, "Vertical accelerations of simple beams due to successive loads traveling at resonant speeds," Journal of Sound and Vibration, vol. 289, no. 1-2, pp. 210-228, 2006.

[6] H. Xia, N. Zhang, and W. W. Guo, "Analysis of resonance mechanism and conditions of train-bridge system," Journal of Sound and Vibration, vol. 297, no. 3-5, pp. 810-822, 2006.

[7] K. Liu, G. De Roeck, and G. Lombaert, "The effect of dynamic train-bridge interaction on the bridge response during a train passage," Journal of Sound and Vibration, vol. 325, no. 1-2, pp. 240-251, 2009.

[8] L. Mao and Y. Lu, "Critical speed and resonance criteria of railway bridge response to moving trains," Journal of Bridge Engineering, vol. 18, no. 2, pp. 131-141, 2013.

[9] Y. B. Yang and J. D. Yau, "Vertical and pitching resonance of train cars moving over a series of simple beams," Journal of Sound and Vibration, vol. 337, pp. 135-149, 2015.

[10] Y. B. Yang and J. D. Yau, "Resonance of high-speed trains moving over a series of simple or continuous beams with nonballasted tracks," Engineering Structures, vol. 143, pp. 295305, 2017.

[11] E. Moliner, M. D. Martínez-Rodrigo, and P. Museros, "Dynamic performance of existing double track railway bridges at resonance with the increase of the operational line speed," Engineering Structures, vol. 132, pp. 98-109, 2017.

[12] Y. F. Duan, S. M. Wang, and J. D. Yau, "Vector form intrinsic finite element method for analysis of train-bridge interaction problems considering the coach-coupler effect," International Journal of Structural Stability and Dynamics, vol. 19, no. 02, Article ID 1950014, 2019.

[13] J. D. Yau, M. D. Martínez-Rodrigo, and A. Doménech, “An equivalent additional damping approach to assess vehiclebridge interaction for train-induced vibration of short-span railway bridges," Engineering Structures, vol. 188, pp. 469-479, 2019.

[14] C. D. Stoura and E. G. Dimitrakopoulos, "Additional damping effect on bridges because of vehicle-bridge interaction," Journal of Sound and Vibration, vol. 476, Article ID 115294, 2020.

[15] W. Zhai, Z. Han, Z. Chen, L. Ling, and S. Zhu, "Train-trackbridge dynamic interaction: a state-of-the-art review," Vehicle System Dynamics, vol. 57, no. 7, pp. 984-1027, 2019.

[16] Z.-w. Yu and J.-f. Mao, "Probability analysis of train-trackbridge interactions using a random wheel/rail contact model," Engineering Structures, vol. 144, pp. 120-138, 2017.

[17] J. Mao, Z. Yu, Y. Xiao, C. Jin, and Y. Bai, "Random dynamic analysis of a train-bridge coupled system involving random system parameters based on probability density evolution method," Probabilistic Engineering Mechanics, vol. 46, pp. 48-61, 2016.

[18] X. Xiao, Y. Yan, and B. Chen, "Stochastic dynamic analysis for vehicle-track-bridge system based on probability density evolution method," Engineering Structures, vol. 188, pp. 745-761, 2019.

[19] S. Wang, J. Luo, S. Zhu, Z. Han, and G. Zhao, "Random dynamic analysis on a high-speed train moving over a longspan cable-stayed bridge," International Journal of Reality Therapy, 2021.

[20] J. Li and J. Chen, Stochastic Dynamic Structures, John Wiley \& Sons(Asia) Pte Ltd, Solaris, Singapore, 2009.

[21] J. Li and J. B. Chen, "Probability density evolution method for dynamic response analysis of structures with uncertain parameters," Computational Mechanics, vol. 34, no. 5, pp. 400-409, 2004.

[22] L. Xu, W. Zhai, and Z. Li, "A coupled model for train-trackbridge stochastic analysis with consideration of spatial variation and temporal evolution," Applied Mathematical Modelling, vol. 63, pp. 709-731, 2018.

[23] L. Xin, X. Li, J. Zhang, Y. Zhu, and L. Xiao, "Resonance analysis of train-track-bridge interaction systems with correlated uncertainties," International Journal of Structural Stability and Dynamics, vol. 20, no. 01, Article ID 2050008, 2020.

[24] W. Zhai, K. Wang, and C. Cai, "Fundamentals of vehicle-track coupled dynamics," Vehicle System Dynamics, vol. 47, no. 11, pp. 1349-1376, 2009.

[25] W.-M. Zhai, "Two simple fast integration methods for largescale dynamic problems in engineering," International Journal for Numerical Methods in Engineering, vol. 39, no. 24, pp. 4199-4214, 1996.

[26] J. Luo, S. Zhu, and W. Zhai, "Theoretical modelling of a vehicle-slab track coupled dynamics system considering longitudinal vibrations and interface interactions," Vehicle System Dynamics, vol. 59, no. 9, pp. 1313-1334, 2021.

[27] J. Luo, S. Zhu, and W. Zhai, "An advanced train-slab track spatially coupled dynamics model: theoretical methodologies and numerical applications," Journal of Sound and Vibration, vol. 501, Article ID 116059, 2021.

[28] J. Luo, S. Zhu, and W. Zhai, "Formulation of curved beam vibrations and its extended application to train-track spatial 
interactions," Mechanical Systems and Signal Processing, vol. 165, Article ID 108393, 2022.

[29] J. Li and J. Chen, "The number theoretical method in response analysis of nonlinear stochastic structures," Computational Mechanics, vol. 39, pp. 693-708, 2007.

[30] L. Hua and Y. Wang, Applications of Number Theory to Numerical Analysis, Springer, Heidelberg Berlin New York, 1981.

[31] European committee for standardization (Cen), EN 1991-2. Eurocode 1: Actions on structures: Traffic Loads on Bridges, Brussels, 2003. 